\title{
Trichotomy of nonoscillatory solutions to second-order neutral difference equation with quasi-difference
}

\author{
Agata Bezubik', Małgorzata Migda², Magdalena Nockowska-Rosiak ${ }^{3}$ and Ewa Schmeidel ${ }^{*}$
}

"Correspondence:

eschmeidel@math.uwb.edu.pl

${ }^{1}$ Institute of Mathematics, University of Bialystok, Ciołkowskiego 1M,

Białystok, 15-245, Poland

Full list of author information is

available at the end of the article

\begin{abstract}
In this paper the nonlinear second-order neutral difference equation of the following form: $\Delta\left(a_{n} \Delta\left(x_{n}-p_{n} x_{n-1}\right)\right)+q_{n} f\left(x_{n-\tau}\right)=0$ is considered. By suitable substitution the above equation is transformed into a new one, which is a third-order non-neutral difference equation. Using results obtained for the new equation, the asymptotic properties of the neutral difference equation are studied. Some classification of nonoscillatory solutions is presented, as well as an estimation of the solutions. Finally, we present necessary and sufficient conditions for the existence of solutions to both considered equations being asymptotically equivalent to the given sequences.
\end{abstract}

MSC: 39A10; 39A22

Keywords: difference equation; second order; neutral type; nonoscillatory solutions; estimation of solutions

\section{Introduction}

In this paper we consider the difference equation in the following form:

$$
\Delta\left(a_{n} \Delta\left(x_{n}-p_{n} x_{n-1}\right)\right)+q_{n} f\left(x_{n-\tau}\right)=0, \quad n \in \mathbb{N}_{\max \{1, \tau\}},
$$

where $\Delta$ is the forward difference operator defined by $\Delta y_{n}=y_{n+1}-y_{n},\left(a_{n}\right),\left(p_{n}\right),\left(q_{n}\right)$ are sequences of positive real numbers, $\tau$ is a nonnegative integer, and the function $f: \mathbb{N} \rightarrow \mathbb{R}$. Here $\mathbb{R}$ is the set of real numbers $\mathbb{N}=\{1,2, \ldots\}$, and $\mathbb{N}_{k}=\{k, k+1, k+2, \ldots\}, k \in \mathbb{N}$.

By a solution to (1) we mean a sequence $\left(x_{n}\right)$ which satisfies (1) for $n$ sufficiently large. We consider only solutions which are nontrivial for all large $n$. A solution to (1) is called nonoscillatory if it is eventually positive or eventually negative. Otherwise it is called oscillatory.

Let us denote

$$
y_{n+1}=x_{n} \prod_{i=1}^{n} \frac{1}{p_{i}} .
$$

This implies that $x_{n}-p_{n} x_{n-1}=\left(\Delta y_{n}\right) \prod_{i=1}^{n} p_{i}$. Substitution of (2) transforms (1) into the following:

$$
\Delta\left(a_{n} \Delta\left(\left(\Delta y_{n}\right) \prod_{i=1}^{n} p_{i}\right)\right)+q_{n} f\left(y_{n-\tau+1} \prod_{i=1}^{n-\tau} p_{i}\right)=0 .
$$

(c) 2015 Bezubik et al. This article is distributed under the terms of the Creative Commons Attribution 4.0 International License (http://creativecommons.org/licenses/by/4.0/), which permits unrestricted use, distribution, and reproduction in any medium, provided you give appropriate credit to the original author(s) and the source, provide a link to the Creative Commons license, and indicate if changes were made. 
Setting

$$
b_{n}=\prod_{i=1}^{n} p_{i}
$$

and assuming that

$$
f\left(b_{n-\tau} z\right)=b_{n}^{*} g(z)
$$

in (3), we get the third-order nonlinear difference equation of the following form:

$$
\Delta\left(a_{n} \Delta\left(b_{n} \Delta y_{n}\right)\right)+q_{n}^{*} g\left(y_{n+1-\tau}\right)=0, \quad n \in \mathbb{N}_{\max \{1, \tau\}},
$$

where

$$
q_{n}^{*}=q_{n} b_{n}^{*}
$$

By virtue of (4), the positivity of terms of the sequence $\left(p_{n}\right)$ implies the positivity of terms of the sequence $\left(b_{n}\right)$. Note that $f(x y)=f(x) f(y)$ is satisfied for all power functions. Hence, by (5) and (7), if $f(x)=x^{\gamma}$, where $\gamma$ is a positive constant, then $g=f$ and $b_{n}^{*}=b_{n-\tau}^{\gamma}$ for all $n \in \mathbb{N}_{\tau}$. If $f$ is not a power function, in some cases we can find the function $g$ assumed by (5). For example, for $f(x)=x^{3} 2^{x-1}$ and $b_{n} \equiv b \in \mathbb{R}$ we have $b_{n}^{*}=\frac{1}{2} b^{3}$ and $g(x)=\left(2^{b}\right)^{x} x^{3}$.

Neutral type difference equations have been widely studied in the literature. Some recent results on the asymptotic behavior of second-order neutral difference equations can be found, for example, in [1-7]. The higher-order neutral difference equations were studied in [8-13].

For results concerning the oscillatory and asymptotic behavior of the third-order difference equation we refer to [14, 15], for equations with quasi-differences to [16-19], and to the references cited therein. Many results on the oscillation of second- and third-order functional differential and difference equations can also be found in [20].

The purpose of this paper is to study the asymptotic properties of the neutral difference equation (1). Transforming the considered equation into a new one, which is a third-order difference equation of type (6), we get various results concerning the asymptotic behavior of solutions to this equation. These results are then used to establish some properties of the solutions to (1). In particular, we obtain necessary and sufficient conditions for the existence of solutions asymptotically equivalent to the given sequences.

Fourth-order non-neutral difference equations with one quasi-difference, by the techniques here used, were studied in [21-23]. Some generalizations of the results presented in these papers were published in $[24,25]$. Even so, there is not a full analogy to the results since the Kneser type classification of the nonoscillatory solution is different for odd- or even-order equations, and of neutral or non-neutral type as well.

Throughout the rest of our investigations, one or several of the following assumptions will be imposed:

$$
\text { (H1) } \sum_{i=1}^{\infty} \frac{1}{a_{i}}=\infty \text {; }
$$


(H2) $\prod_{i=1}^{n} p_{i}=O(n)$;

(H3) $\quad z f(z)>0 \quad$ for all $z \neq 0$;

(H4) $f: \mathbb{R} \rightarrow \mathbb{R}$ is a continuous function.

Notice that, by virtue of $(5)$, the positivity of the sequence $\left(b_{n}\right)$ implies that conditions (H3) and (H4) hold also for the function $g$.

The following definitions and theorems will be used in the sequel.

We say that the sequence $\left(u_{n}\right)$ is asymptotically constant if this sequence has a nonzero limit, and we say that it is an asymptotically zero sequence if the limit of this sequence equals zero. We say that the sequence $\left(u_{n}\right)$ is asymptotically equivalent to $\left(v_{n}\right)$ if $\left(\frac{u_{n}}{v_{n}}\right)$ has a nonzero limit. In the present paper, we study the three types of solutions: asymptotically zero solutions, asymptotically constant solutions, and unbounded solutions. It is called a trichotomy of nonoscillatory solutions.

Definition 1 (Uniformly Cauchy subset [26]) A subset $S$ of the Banach space $B$ is said to be uniformly Cauchy if for every $\varepsilon>0$ there exists a positive integer $N$ such that $\left|x_{i}-x_{j}\right|<\varepsilon$ whenever $i, j>N$ for any $\left(x_{n}\right) \in B$.

Lemma 1 (Arzela-Ascoli's theorem [26]) Each bounded and uniformly Cauchy subset of $B$ is relatively compact.

Theorem 1 (Schauder theorem [27]) Let $S$ be a nonempty, closed, and convex subset of a Banach space $B$ and $T: S \rightarrow S$ be a continuous mapping such that $T(S)$ is a relatively compact subset of $B$. Then $T$ has at least one fixed point in $S$.

The following theorem of Stolz-Cesáro is a discrete analog of l'Hospital's rule.

Theorem 2 (Stolz-Cesáro theorem [28]) Let $\left(u_{n}\right),\left(v_{n}\right)$ be two sequences of real numbers. Assume that $\left(v_{n}\right)$ is a strictly monotone and divergent sequence, and the following limit exists: $\lim _{n \rightarrow \infty} \frac{\Delta u_{n}}{\Delta v_{n}}=g$. Then

$$
\lim _{n \rightarrow \infty} \frac{u_{n}}{v_{n}}=g
$$

We introduce the following notation:

$$
Q_{n}=\sum_{k=1}^{n-1} \frac{1}{b_{k}} \sum_{j=1}^{k-1} \frac{1}{a_{j}}=\sum_{j=1}^{n-1} \frac{1}{a_{j}} \sum_{k=j+1}^{n-1} \frac{1}{b_{k}} .
$$

\section{Existence of nonoscillatory solutions}

In this section, we obtain necessary and sufficient conditions for the existence of nonoscillatory solutions to (1) with certain asymptotic properties. We start with the following lemmas. 
Lemma 2 Condition (H2) implies that

$$
\sum_{i=1}^{\infty} \frac{1}{b_{i}}=\infty,
$$

where $\left(b_{n}\right)$ is defined by (4).

Proof Condition (H2) implies that $\prod_{i=1}^{n} p_{i} \leq C_{0} n$, where $C_{0}$ is a positive constant. It follows that $\prod_{i=1}^{n} p_{i}^{-1} \geq \frac{1}{C_{0} n}$. Using the notation of (4), the above inequality takes the form $\frac{1}{b_{n}} \geq$ $\frac{1}{C_{0} n}$. Since the series $\sum_{n=1}^{\infty} \frac{1}{n}$ diverges, condition (9) is satisfied.

Remark 1 Condition (H1) and (9) imply that

$$
\lim _{n \rightarrow \infty} Q_{n}=\infty,
$$

where $\left(Q_{n}\right)$ is defined by $(8)$.

Lemma 3 Assume that (H1), (H2), and the following conditions:

$\left(\mathrm{H}^{*} 3\right) \quad z g(z)>0 \quad$ for all $z \neq 0$;

$\left(\mathrm{H}^{*} 4\right) \quad g: \mathbb{R} \rightarrow \mathbb{R}$ is a continuous function;

are satisfied. Let $\left(y_{n}\right)$ be an eventually positive solution to (6). Then exactly one of the following statements holds:

(i) $y_{n}>0, \quad \Delta y_{n}>0, \quad \Delta\left(b_{n} \Delta y_{n}\right)>0$,

(ii) $y_{n}>0, \quad \Delta y_{n}<0, \quad \Delta\left(b_{n} \Delta y_{n}\right)>0$

for all sufficiently large $n$.

Proof The proof is obvious and hence omitted.

Lemma 4 Assume that (H1)-(H4) hold. If $\left(x_{n}\right)$ is an eventually positive solution to (1), then exactly one of the following cases holds:

(I)

$$
\lim _{n \rightarrow \infty} \frac{x_{n}}{b_{n}}=0
$$

(II) there exist positive constants $C_{1}, C_{2}$, and a positive integer $n_{0}$ such that

$$
C_{1} b_{n} \leq x_{n} \leq C_{2} b_{n} Q_{n+1} \quad \text { for } n \geq n_{0},
$$

where $\left(b_{n}\right)$ is defined by $(4)$ and $\left(Q_{n}\right)$ is defined by (8).

Proof Let $\left(y_{n}\right)$ be an eventually positive solution to (6). Then, by Lemma 3, we have two possibilities:

$$
\lim _{n \rightarrow \infty} y_{n}=0
$$

or there exists a positive constant $C_{1}$ such that $y_{n} \geq C_{1}$. 
If $\lim _{n \rightarrow \infty} y_{n}=0$, then condition (I) is satisfied.

Assuming that $y_{n+1} \geq C_{1}$ and using substitution (2), we obtain

$$
x_{n} \prod_{i=1}^{n} p_{i}^{-1} \geq C_{1} \text {. }
$$

Thus, inequality $C_{1} b_{n} \leq x_{n}$ from (11) is satisfied.

Next, we prove that in case (II) the inequality $x_{n} \leq C_{2} b_{n} Q_{n+1}$ is also satisfied. Since (H3) is satisfied for the function $g$, from the point of view of (6), there exists $n_{1}$ such that

$$
\Delta\left(a_{n} \Delta\left(b_{n} \Delta y_{n}\right)\right)<0 \quad \text { for } n \geq n_{1} .
$$

By Lemma 2, if (H1) and (H2) are satisfied, then there exists $n_{2} \geq n_{1}$ such that

$$
1 \leq \sum_{i=n_{2}}^{n-1} \frac{1}{b_{i}} \leq \sum_{i=n_{2}}^{n-1} \frac{1}{b_{i}} \sum_{j=n_{2}}^{i-1} \frac{1}{a_{j}} \quad \text { for } n \geq n_{2} .
$$

Summing inequality (12) from $n_{2}$ to $n-1$, we get

$$
\Delta\left(b_{n} \Delta y_{n}\right)<\frac{A_{1}}{a_{n}} \quad \text { for } n \geq n_{2}
$$

where $A_{1}=a_{n_{2}} \Delta\left(b_{n_{2}} \Delta y_{n_{2}}\right)$ is a positive constant. Summing again, we have

$$
b_{n} \Delta y_{n}<A_{1} \sum_{i=n_{2}}^{n-1} \frac{1}{a_{i}}+A_{2} \text {, }
$$

where $A_{2}=\max \left\{0, b_{n_{2}} \Delta y_{n_{2}}\right\}$ is a nonnegative constant. Therefore

$$
\Delta y_{n}<\frac{A_{1}}{b_{n}} \sum_{i=n_{2}}^{n-1} \frac{1}{a_{i}}+\frac{A_{2}}{b_{n}} \quad \text { for } n \geq n_{2}+1 .
$$

Summing again, we have

$$
y_{n}<A_{1} \sum_{j=n_{2}}^{n-1} \frac{1}{b_{j}} \sum_{l=n_{2}}^{j-1} \frac{1}{a_{i}}+A_{2} \sum_{j=n_{2}}^{n-1} \frac{1}{b_{j}}+A_{3}, \quad n \geq n_{2}+2,
$$

where $A_{3}=y_{n_{2}}$ is a positive constant.

By (13), it is easy to see that each term on the right side of inequality (14) is less than

$$
\max \left\{A_{1}, A_{2}, A_{3}\right\} \sum_{j=n_{2}}^{n-1} \frac{1}{b_{j}} \sum_{i=n_{2}}^{j-1} \frac{1}{a_{i}} .
$$

From (14), we get

$$
y_{n} \leq C_{2} \sum_{j=n_{2}}^{n-1} \frac{1}{b_{j}} \sum_{i=n_{2}}^{j-1} \frac{1}{a_{i}} \quad \text { for sufficiently large } n,
$$


where $C_{2}=3 \max \left\{A_{1}, A_{2}, A_{3}\right\}$. Hence

$$
y_{n+1} \leq C_{2} \sum_{j=n_{2}}^{n} \frac{1}{b_{j}} \sum_{i=n_{2}}^{j-1} \frac{1}{a_{i}} \text { for sufficiently large } n .
$$

Using the substitutions (2) and (4), we obtain

$$
\frac{x_{n}}{b_{n}} \leq C_{2} \sum_{j=n_{2}}^{n} \frac{1}{b_{j}} \sum_{i=n_{2}}^{j-1} \frac{1}{a_{i}} .
$$

By (8), we see that the required inequality is proved.

As a consequence of Lemma 4 we obtain the following result.

Lemma 5 Assume that $(\mathrm{H} 1),(\mathrm{H} 2),\left(\mathrm{H}^{*} 3\right)$, and $\left(\mathrm{H}^{*} 4\right)$ hold. If $\left(y_{n}\right)$ is an eventually positive solution to (6), then

(I)

$$
\lim _{n \rightarrow \infty} y_{n}=0
$$

(II) there exist positive constants $C_{1}$ and $C_{2}$ such that

$$
C_{1} \leq y_{n} \leq C_{2} Q_{n} \text { for large } n
$$

Before we derive a necessary and sufficient condition for the existence of a solution to (1) that is asymptotically equivalent to $\left(b_{n}\right)$, the following theorem needs to be proved.

Theorem 3 Let conditions (H1), (H2), ( $\left.\mathrm{H}^{*} 3\right),\left(\mathrm{H}^{*} 4\right)$ be satisfied. Then a necessary condition for (6) to have an asymptotically constant solution is that

$$
\sum_{i=1}^{\infty} \frac{1}{b_{i}} \sum_{j=i}^{\infty} \frac{1}{a_{j}} \sum_{k=j}^{\infty} q_{k}^{*}<\infty .
$$

Proof Let $\left(y_{n}\right)$ be an asymptotically constant solution to (6). Then $\left(y_{n}\right)$ is a nonoscillatory sequence. Without loss of generality, we assume that $\left(y_{n}\right)$ is an eventually positive solution. By Lemma 3 it is of type (i) or type (ii). Each solution to type (i) tends to infinity. This implies that $\left(y_{n}\right)$ is of type (ii).

Let us denote

$$
\lim _{n \rightarrow \infty} y_{n}=\alpha>0 .
$$

Then there exist positive constants $C_{3}$ and $C_{4}$ such that

$$
C_{3} \leq y_{n+1-\tau} \leq C_{4} \quad \text { for large } n \text {. }
$$

By $\left(\mathrm{H}^{*} 3\right)$ and $\left(\mathrm{H}^{*} 4\right)$, we see that there exists a positive constant

$$
C_{5}=\min _{z \in\left[C_{3}, C_{4}\right]}\{g(z)\},
$$


which means that, for $y_{n+1-\tau} \in\left[C_{3}, C_{4}\right]$, we have

$$
C_{5} \leq g\left(y_{n+1-\tau}\right) \quad \text { for large } n
$$

Let $n_{3}$ be so large that (17) and (ii) are satisfied for $n \geq n_{3}$. Next, we rewrite (6) in the form

$$
-\Delta\left(a_{i} \Delta\left(b_{i} \Delta y_{i}\right)\right)=q_{i}^{*} g\left(y_{i+1-\tau}\right)
$$

Multiplying the above equation by $\sum_{j=n_{3}}^{i} \frac{1}{a_{j}} \sum_{k=n_{3}}^{j} \frac{1}{b_{k}}$ and summing both sides of it from $i=n_{3}-2$ to $n-2$ we obtain

$$
\begin{gathered}
-\sum_{i=n_{3}-2}^{n-2}\left(\sum_{j=n_{3}}^{i} \frac{1}{a_{j}} \sum_{k=n_{3}}^{j} \frac{1}{b_{k}}\right) \Delta\left(a_{i} \Delta\left(b_{i} \Delta y_{i}\right)\right) \\
=\sum_{i=n_{3}-2}^{n-2} q_{i}^{*} g\left(y_{i+1-\tau}\right)\left(\sum_{j=n_{3}}^{i} \frac{1}{a_{j}} \sum_{k=n_{3}}^{j} \frac{1}{b_{k}}\right) .
\end{gathered}
$$

By (17), the following inequality holds:

$$
\sum_{i=n_{3}-2}^{n-2} q_{i}^{*} g\left(y_{i+1-\tau}\right)\left(\sum_{j=n_{3}}^{i-1} \frac{1}{a_{j}} \sum_{k=n_{3}}^{j} \frac{1}{b_{k}}\right) \geq C_{5} \sum_{i=n_{3}-2}^{n-2} q_{i}^{*}\left(\sum_{j=n_{3}}^{i} \frac{1}{a_{j}} \sum_{k=n_{3}}^{j} \frac{1}{b_{k}}\right)
$$

By the formula $\sum_{i=N}^{n-2} y_{i} \Delta x_{i}=\left.x_{i} y_{i}\right|_{i=N} ^{n-1}-\sum_{i=N}^{n-2} x_{i+1} \Delta y_{i}$, we get

$$
\begin{aligned}
- & \sum_{i=n_{3}-2}^{n-2}\left(\sum_{j=n_{3}}^{i} \frac{1}{a_{j}} \sum_{k=n_{3}}^{j} \frac{1}{b_{k}}\right)\left(\Delta\left(a_{i} \Delta\left(b_{i} \Delta y_{i}\right)\right)\right) \\
= & -\left.\left(\sum_{j=n_{3}}^{i} \frac{1}{a_{j}} \sum_{k=n_{3}}^{j} \frac{1}{b_{k}}\right)\left(a_{i} \Delta\left(b_{i} \Delta y_{i}\right)\right)\right|_{i=n_{3}-2} ^{n-1} \\
& +\sum_{i=n_{3}-2}^{n-2}\left(\frac{1}{a_{i+1}} \sum_{k=N}^{i+1} \frac{1}{b_{k}}\right)\left(a_{i+1} \Delta\left(b_{i+1} \Delta y_{i+1}\right)\right) \\
< & \sum_{i=n_{3}-2}^{n-2}\left(\sum_{k=n_{3}}^{i+1} \frac{1}{b_{k}}\right)\left(\Delta\left(b_{i+1} \Delta y_{i+1}\right)\right) \\
= & \left.\left(\sum_{k=n_{3}}^{i+1} \frac{1}{b_{k}}\right)\left(b_{i+1} \Delta y_{i+1}\right)\right|_{i=n_{3}-2} ^{n-1} \sum_{i=n_{3}-2}^{n-2}\left(\frac{1}{b_{i+2}}\right)\left(b_{i+2} \Delta y_{i+2}\right) \\
< & -\sum_{i=n_{3}-2}^{n-2} \Delta y_{i+2}=y_{n_{3}}-y_{n+1},
\end{aligned}
$$

which tends to $y_{n_{3}}-\alpha$ where $\alpha$ is defined by (16). Since $\left(y_{n}\right)$ is a decreasing sequence we have $y_{n_{3}}-\alpha>0$. Set $C_{6}=y_{n_{3}}-\alpha$. From the above, (18), and (19) we get

$$
C_{5} \sum_{i=n_{3}-2}^{\infty} q_{i}^{*}\left(\sum_{j=n_{3}}^{i} \frac{1}{a_{j}} \sum_{k=n_{3}}^{j} \frac{1}{b_{k}}\right)<C_{6}
$$


This means that

$$
\sum_{i=1}^{\infty} q_{i}^{*} \sum_{j=1}^{i} \frac{1}{a_{j}} \sum_{k=1}^{j} \frac{1}{b_{k}}<\infty .
$$

The above condition is equivalent to condition (15).

The next example shows that the condition (15) in Theorem 3 is not a necessary condition for (6) to have an asymptotically zero solution.

Example 1 Let us consider the following equation of the form (6):

$$
\Delta^{3} y_{n}+\frac{1}{8} y_{n}=0
$$

Here $a_{n} \equiv 1, b_{n} \equiv 1, q_{n}^{*} \equiv \frac{1}{8}, g(x)=x$, and $\tau=1$. It is easy to see that condition (15) is not satisfied, but the above equation has an asymptotically zero solution $y_{n}=\frac{1}{2^{n}}$.

Sufficient conditions, under which, for every real constant, there exists a solution to the higher-order difference equation with quasi-differences convergent to this constant are obtained in Theorem 3.3 in [29]. Hence, for (6), we have the following.

Theorem 4 Assume that $(\mathrm{H} 1),(\mathrm{H} 2),\left(\mathrm{H}^{*} 3\right),\left(\mathrm{H}^{*} 4\right)$ hold and condition $(15)$ is satisfied. Then for every $c \in \mathbb{R}$ there exists a solution $x$ to (1) such that $\lim _{n \rightarrow \infty} x(n)=c$.

Corollary 1 Let conditions (H1), (H2), $\left(\mathrm{H}^{*} 3\right),\left(\mathrm{H}^{*} 4\right)$ be satisfied. Then the condition

$$
\sum_{i=1}^{\infty} \frac{1}{b_{i}} \sum_{j=i}^{\infty} \frac{1}{a_{j}} \sum_{k=j}^{\infty} q_{k}^{*}=\infty
$$

implies that (6) has no asymptotically constant solution.

Proof This corollary follows directly from Theorem 3.

Theorem 5 If conditions ( $\mathrm{H1})-(\mathrm{H} 4)$ are satisfied, then a necessary and sufficient condition for (1) to have a solution $\left(x_{n}\right)$ asymptotically equivalent to the sequence $\left(\prod_{i=1}^{n} p_{i}\right)$ is the condition

$$
\sum_{i=1}^{\infty} \prod_{l=1}^{i} \frac{1}{p_{l}} \sum_{j=i}^{\infty} \frac{1}{a_{j}} \sum_{k=j}^{\infty} q_{k} b_{k}^{*}<\infty .
$$

Proof Using the notation of (2), (5), and (7) in condition (15) the conclusion of this theorem follows directly from Theorem 3 and Theorem 4 .

Remark 2 Let the assumptions of Theorem 5 be satisfied. If

$$
\lim _{n \rightarrow \infty} \prod_{i=1}^{n} p_{i}=0,
$$


then condition (21) is a necessary and sufficient condition for (1) to have an asymptotically zero solution such that $\left(x_{n}\right) \sim\left(\prod_{i=1}^{n} p_{i}\right)$.

As a consequence of Theorem 5 we get the following result for the Emden-Fowler type equation

$$
\Delta\left(a_{n} \Delta\left(x_{n}-p_{n} x_{n-1}\right)\right)+q_{n} x_{n-\tau}^{\gamma}=0, \quad n \in \mathbb{N}_{\max \{1, \tau\}},
$$

where $\left(a_{n}\right),\left(p_{n}\right),\left(q_{n}\right)$ are sequences of positive real numbers, $\tau$ is a nonnegative integer, and $\gamma$ is the ratio of odd positive integers.

Corollary 2 Let conditions (H1) and (H2) be satisfied. A necessary and sufficient condition for (22) to have a solution $\left(x_{n}\right)$ asymptotically equivalent to the sequence $\left(\prod_{i=1}^{n} p_{i}\right)$ is the condition

$$
\sum_{i=1}^{\infty} \prod_{l=1}^{i} \frac{1}{p_{l}} \sum_{j=i}^{\infty} \frac{1}{a_{j}} \sum_{k=j}^{\infty} q_{k} \prod_{l=1}^{k-\tau} p_{l}^{\gamma}<\infty .
$$

Example 2 Consider the following equation:

$$
\begin{gathered}
\Delta\left(\left(\sqrt{\frac{n+1}{n}}+\sqrt{\frac{n+2}{n+1}}\right) \Delta\left(x_{n}-\sqrt{\frac{n+1}{n}} x_{n-1}\right)\right) \\
+\frac{2}{n(n+1)(n+2)(\sqrt{n}+1)^{3}} x_{n-1}^{3}=0, \quad n \in \mathbb{N}_{1} .
\end{gathered}
$$

Here $a_{n}=\sqrt{\frac{n+1}{n}}+\sqrt{\frac{n+2}{n+1}}, p_{n}=\sqrt{\frac{n+1}{n}}, q_{n}=\frac{2}{n(n+1)(n+2)(\sqrt{n}+1)^{3}}, \gamma=3$, and $\tau=1$. All assumptions of Corollary 2 are satisfied. Hence (24) has at least one solution asymptotically equivalent to the sequence $\left(\prod_{i=1}^{n} p_{i}\right)=\sqrt{n+1}$. In fact $x_{n}=\sqrt{n+1}+1$ is such solution.

Example 3 Consider the following equation:

$$
\Delta^{2}\left(x_{n}-x_{n-1}\right)+\frac{1}{2^{\frac{2}{3} n+\frac{10}{3}}\left(2^{n-1}+1\right)^{\frac{1}{3}}} x_{n-2}^{\frac{1}{3}}=0, \quad n \in \mathbb{N}_{2} .
$$

Here $a_{n} \equiv 1, p_{n} \equiv 1, q_{n}=\frac{1}{2^{\frac{2}{3} n+\frac{10}{3}}\left(2^{n-1}+1\right)^{\frac{1}{3}}}, \gamma=\frac{1}{3}$, and $\tau=2$. It is easy to check that all assumptions of Corollary 2 are satisfied. Hence, (25) has at least one solution asymptotically equivalent to the sequence $\left(\prod_{i=1}^{n} p_{i}\right) \equiv 1$. This means that (25) has an asymptotically constant solution. In fact $x_{n}=1+\frac{1}{2^{n+1}}$ is one such solution.

Finally, we present a necessary and sufficient condition for the existence of an asymptotically $\left(Q_{n}\right)$ solution to $(1)$. We start with the following theorem.

Theorem 6 If conditions (H1), (H2), $\left(\mathrm{H}^{*} 3\right),\left(\mathrm{H}^{*} 4\right)$ are satisfied and

$$
g \text { is a monotonic function, }
$$

then a necessary and sufficient condition for (6) to have a solution $\left(y_{n}\right)$ satisfying

$$
\lim _{n \rightarrow \infty} \frac{y_{n}}{Q_{n}} \neq 0
$$


is that

$$
\sum_{i=1}^{\infty} q_{i}^{*}\left|g\left(C Q_{i+1-\tau}\right)\right|<\infty
$$

where $C$ is some nonzero constant.

Proof Necessity. Let $\left(y_{n}\right)$ be a nonoscillatory solution to (6) which satisfies (27). Without loss of generality, we may assume that

$$
\lim _{n \rightarrow \infty} \frac{y_{n}}{Q_{n}}=\beta>0 .
$$

Then there exist positive constants $C_{7}$ and $C_{8}$ such that

$$
C_{7} Q_{n} \leq y_{n} \leq C_{8} Q_{n} \quad \text { for large } n .
$$

Hence

$$
C_{7} Q_{n+1-\tau} \leq y_{n+1-\tau} \leq C_{8} Q_{n+1-\tau} \quad \text { for large } n \text {, say } n \geq n_{4} .
$$

Thus, by (26), we get

$$
g\left(y_{n+1-\tau}\right) \geq g\left(C_{9} Q_{n+1-\tau}\right),
$$

where $C_{9}=C_{7}$ if the function $g$ is nondecreasing and $C_{9}=C_{8}$ if the function $g$ is nonincreasing.

By $\left(\mathrm{H}^{*} 3\right)$, we see that $g\left(C_{9} Q_{n+1-\tau}\right)$ is positive. On the other hand, summing (6) from $n_{5}=n_{4}+\tau$ to $n-1$, by Lemma 3 , we obtain

$$
0<a_{n} \Delta\left(b_{n} \Delta y_{n}\right)=a_{n_{5}} \Delta\left(b_{n_{5}} \Delta y_{n_{5}}\right)-\sum_{i=n_{5}}^{n-1} q_{i}^{*} g\left(y_{i+1-\tau}\right) \quad \text { for } n \geq n_{5} .
$$

This implies that

$$
\sum_{i=n_{5}}^{n-1} q_{i}^{*} g\left(y_{i+1-\tau}\right) \leq a_{n_{5}} \Delta\left(b_{n_{5}} \Delta y_{n_{5}}\right)<\infty
$$

So, by (29), we have

$$
\sum_{i=n_{5}}^{\infty} q_{i}^{*} g\left(C_{9} Q_{n+1-\tau}\right)<\infty
$$

Sufficiency. Let $C_{10}$ be a given positive constant. Set

$$
I_{n}=\left[\frac{C_{10}}{2} Q_{n}, C_{10} Q_{n}\right] .
$$


From the above, (26) and $\left(\mathrm{H}^{*} 4\right)$, there exists a maximum of the function $g$ on interval $I_{n}$, which we denote as the point $C_{11} Q_{n}$ with $C_{11}=\frac{C_{10}}{2}$ if the function $g$ is nonincreasing and $C_{11}=C_{10}$ if the function $g$ is nondecreasing. Thus we get

$$
g\left(y_{n}\right) \leq g\left(C_{11} Q_{n}\right) \quad \text { for } n \in I_{n} .
$$

Assume that (28) holds for $C=C_{11}$. Then there exists a positive integer $n_{6}$ such that

$$
\sum_{i=n_{6}}^{\infty} q_{i}^{*} g\left(C Q_{n+1-\tau}\right) \leq \frac{C_{10}}{2} .
$$

Consider the Banach space $B$ of all real sequences $y=\left(y_{n}\right)$ such that

$$
\|y\|=\sup _{n \geq n 7} \frac{\left|y_{n}\right|}{Q_{n}^{2}}<\infty
$$

where $n_{7}=n_{6}+\tau-1$. We have

$$
S=\left\{\left(y_{n}\right) \in B: y_{n}=\frac{C_{10}}{2} \text { for } n<n_{7}, y_{n} \in I_{n} \text { for } n \geq n_{7}\right\} .
$$

It is easy to see that $S$ is a bounded, convex and closed subset of $B$.

Now we define an operator $T: S \rightarrow B$ in the following way:

$$
(T y)_{n}= \begin{cases}\frac{C_{10}}{2} Q_{n} & \text { for } n<n_{7}, \\ \frac{C_{10}}{2} Q_{n}+\sum_{k=n_{7}}^{n-1} \frac{1}{b_{k}} \sum_{j=n_{7}}^{k-1} \frac{1}{a_{j}} \sum_{i=j}^{\infty} q_{i}^{*} g\left(y_{i+1-\tau}\right) & \text { for } n \geq n_{7} .\end{cases}
$$

First we show that $T(S) \subset S$. Indeed, if $y \in S$ it is clear from (32) that $(T y)_{n} \geq \frac{C_{10}}{2} Q_{n}$ for $n \geq 1$. Furthermore, by (30), we have

$$
\begin{aligned}
(T y)_{n} & <\frac{C_{10}}{2} Q_{n}+\sum_{k=n 7}^{n-1} \frac{1}{b_{k}} \sum_{j=n 7}^{k-1} \frac{1}{a_{j}} \sum_{i=j}^{\infty} q_{i}^{*} g\left(y_{i+1-\tau}\right) \\
& <\frac{C_{10}}{2} Q_{n}+\sum_{k=1}^{n-1} \frac{1}{b_{k}} \sum_{j=1}^{k-1} \frac{1}{a_{j}} \sum_{i=n_{7}}^{\infty} q_{i}^{*} g\left(y_{i+1-\tau}\right) \\
& \leq \frac{C_{10}}{2} Q_{n}+Q_{n} \sum_{i=n_{7}}^{\infty} q_{i}^{*} g\left(C Q_{n+1-\tau}\right) \\
& \leq \frac{C_{10}}{2} Q_{n}+Q_{n} \frac{C_{10}}{2}=C_{10} Q_{n} .
\end{aligned}
$$

Thus $T$ maps $S$ into itself.

Next we prove that $T$ is continuous. Let $\left(y^{(m)}\right)$ be a sequence in $S$ such that $y^{(m)} \rightarrow y$ as $m \rightarrow \infty$. Because $S$ is closed, $y \in S$. Now, we get

$$
\left|\left(T y^{(m)}\right)_{n}-(T y)_{n}\right| \leq Q_{n} \sum_{i=n_{7}}^{\infty} q_{i}^{*}\left|g\left(y_{i+1-\tau}^{(m)}\right)-g\left(y_{i+1-\tau}\right)\right|
$$


and therefore

$$
\left\|\left(T y^{(m)}\right)_{n}-(T y)_{n}\right\| \leq \frac{1}{Q_{n}} \sum_{i=n_{7}}^{\infty} q_{i}^{*}\left|g\left(y_{i+1-\tau}^{(m)}\right)-g\left(y_{i+1-\tau}\right)\right| .
$$

By (10), (28), and (30), it implies that

$$
\left\|\left(T y^{(m)}\right)_{n}-(T y)_{n}\right\| \leq \frac{2}{Q_{n}} \sum_{i=n 7}^{\infty} q_{i}^{*} g\left(C Q_{i+1-\tau}\right) \rightarrow 0
$$

We see that $T$ is a continuous mapping.

Finally, we need to show that $T(S)$ is uniformly Cauchy. To see this, we have to show that, given any $\varepsilon>0$, there exists an integer $n_{8}$ such that for $m>n>n_{8}$; we have

$$
\left|\frac{(T y)_{m}}{Q_{m}^{2}}-\frac{(T y)_{n}}{Q_{n}^{2}}\right|<\varepsilon
$$

for any $y \in S$. Indeed, we have

$$
\left|\frac{(T y)_{m}}{Q_{m}^{2}}-\frac{(T y)_{n}}{Q_{n}^{2}}\right| \leq \frac{2}{Q_{n}} \sum_{i=1}^{\infty} q_{i}^{*} g\left(y_{i+1-\tau}\right) \leq \frac{C}{Q_{n}} \rightarrow 0 .
$$

Therefore, by Theorem 1 , there exists $y \in S$ such that $y_{n}=(T y)_{n}$ for $n \geq n_{7}$. It is easy to see that $\left(y_{n}\right)$ is a solution to (6).

Furthermore, by Stolz's theorem (see Theorem 2) and (8), we have

$$
\begin{aligned}
\lim _{n \rightarrow \infty} \frac{y_{n}}{Q_{n}} & =\lim _{n \rightarrow \infty} \frac{\Delta y_{n}}{\Delta Q_{n}}=\lim _{n \rightarrow \infty} \frac{b_{n} \Delta y_{n}}{\sum_{j=1}^{n-1} \frac{1}{a_{j}}}=\lim _{n \rightarrow \infty} \frac{\Delta\left(b_{n} \Delta y_{n}\right)}{\Delta\left(\sum_{j=1}^{n-1} \frac{1}{a_{j}}\right)} \\
& =\lim _{n \rightarrow \infty} a_{n} \Delta\left(b_{n} \Delta y_{n}\right) .
\end{aligned}
$$

Thus

$$
\begin{aligned}
\lim _{n \rightarrow \infty} \frac{y_{n}}{Q_{n}} & \leq \lim _{n \rightarrow \infty}\left(\frac{C}{2}+\sum_{i=n}^{\infty} q_{i}^{*} g\left(y_{i+1-\tau}\right)\right) \\
& \leq \lim _{n \rightarrow \infty}\left(\frac{C}{2}+\sum_{i=n}^{\infty} q_{i}^{*} g\left(C_{10} Q_{i+1-\tau}\right)\right)=\frac{C}{2} .
\end{aligned}
$$

This completes the proof.

Remark 3 Note that if the sequences $\left(\frac{1}{a_{n}}\right)$ and $\left(\frac{1}{b_{n}}\right)$ are both polynomial sequences, then $\left(Q_{n}\right)$ is a polynomial sequence, too.

For example, let $\frac{1}{a_{n}}=n$ and $\frac{1}{b_{n}}=n$. Hence $\left(Q_{n}\right)$, defined by (8), takes the following form:

$$
Q_{n}=\sum_{k=1}^{n-1} k \sum_{j=1}^{k-1} j=\frac{1}{2} \sum_{k=1}^{n-1}\left(k^{3}-k^{2}\right)=\frac{(n-1)^{4}}{8}+\frac{(n-1)^{3}}{12}-\frac{(n-1)^{2}}{4}-\frac{n-1}{12} \text {. }
$$

So, $\left(Q_{n}\right)$ is a quartic polynomial. 
Now, let $\frac{1}{a_{n}} \equiv 1$ and $\frac{1}{b_{n}} \equiv 1$. This means that $a_{n} \equiv 1$ and $b_{n} \equiv 1$. Hence $Q_{n}=\frac{1}{2} n^{2}-\frac{3}{2} n+1$ is a quadratic polynomial. Obviously, by virtue of (9), this case holds only if $p_{n} \equiv 1$.

Theorem 7 Let conditions (H1)-(H4) be satisfied and

\section{$f$ is a monotonic function.}

Then a necessary and sufficient condition for (1) to have a solution $\left(x_{n}\right)$ which is asymptotically equivalent to the sequence $\left(Q_{n+1} \prod_{i=1}^{n} p_{i}\right)$ is the convergence of the series

$$
\sum_{i=1}^{\infty} q_{i}\left|C f\left(Q_{i+1-\tau} \prod_{j=1}^{i} p_{j}\right)\right|
$$

where $C$ is some nonzero constant.

Proof Using the notation of (2), (5), and (7) in condition (28) the conclusion of this theorem follows directly from Theorem 6.

Note that for particular cases of $(1)$, if $\left(\frac{1}{a_{n}}\right)$ is a polynomial sequence and $p_{n} \equiv 1$, from Theorem 7 we get the existence of asymptotically polynomial solutions.

Example 4 In Example $3(25)$ is considered. In this equation $a_{n} \equiv 1$ and $p_{n} \equiv 1$. All assumptions of Theorem 7 are satisfied. Hence (25) has an asymptotically $\left(Q_{n}\right)$ solution, where $Q_{n}=\frac{1}{2} n^{2}-\frac{3}{2} n+1$. It means that (25) has an asymptotically polynomial solution.

Some results concerning asymptotically polynomial solutions to difference equations can be found, for example, in [30-34].

\section{Competing interests}

The authors declare that they have no competing interests.

\section{Authors' contributions}

The authors declare that the study was realized in collaboration with the same responsibility. All authors read and approved the final manuscript.

\section{Author details}

${ }^{1}$ Institute of Mathematics, University of Bialystok, Ciołkowskiego 1M, Białystok, 15-245, Poland. ${ }^{2}$ Institute of Mathematics, Poznan University of Technology, Piotrowo 3A, Poznań, 60-965, Poland. ${ }^{3}$ Institute of Mathematics, Lodz University of Technology, Wólczanska 215, Łódź, 90-924, Poland.

\section{Acknowledgements}

This work was partially supported by the Ministry of Science and Higher Education of Poland (PB-43-081/14DS).

Received: 13 February 2015 Accepted: 8 June 2015 Published online: 23 June 2015

\section{References}

1. Galewski, M, Jankowski, R, Nockowska-Rosiak, M, Schmeidel, E: On the existence of bounded solutions for nonlinear second order neutral nonlinear difference equations. Electron. J. Qual. Theory Differ. Equ. 2014, 72 (2014)

2. Galewski, M, Schmeidel, E: On the well posed solutions for nonlinear second order neutral difference equations. Math. Slovaca (2015, in press)

3. Jankowski, R, Schmeidel, E: Almost oscillatory solutions of second order neutral difference equations of neutral type. In: Hartung, F, Pituk, M (eds.) Recent Advances in Delay Differential and Difference Equations. Springer Proceedings in Mathematics and Statistics, vol. 94, pp. 121-130. Springer, Berlin (2014). doi:10.1007/978-3-319-08251-6_4

4. Liu, Z, Xu, Y, Kang, SM: Global solvability for a second order nonlinear neutral delay difference equation. Comput. Math. Appl. 57, 587-595 (2009)

5. Migda, J, Migda, M: Asymptotic properties of solutions of second-order neutral difference equations. Nonlinear Anal. 63, e789-e799 (2005) 
6. Schmeidel, E: Asymptotic behavior of certain second order nonlinear difference equations. In: Elaydi, S, Ladas, G, Aulbach, B, Došly, O (eds.) Proceedings of the Eighth International Conference on Difference Equations and Applications (Brno, 2003), pp. 245-252. Chapman \& Hall/CRC, Boca Raton (2005). doi:10.1201/9781420034905.ch28

7. Schmeidel, E: An application of measures of noncompactness in investigation of boundedness of solutions of second order neutral difference equations. Adv. Differ. Equ. 2013, 91 (2013). doi:10.1186/1687-1847-2013-91

8. Huang, X, Xu, Z: Nonoscillatory solutions of certain higher order neutral difference equations. Southeast Asian Bull. Math. 32, 445-458 (2008)

9. Jankowski, R, Schmeidel, E: Asymptotically zero solution of a class of higher nonlinear neutral difference equations with quasidifferences. Discrete Contin. Dyn. Syst., Ser. B 19, 2691-2696 (2014)

10. Liu, M, Guo, Z: Solvability of a higher-order nonlinear neutral delay difference equation. Adv. Differ. Equ. 2010, Article ID 767620 (2010). doi:10.1155/2010/767620

11. Migda, J, Migda, M: Oscillatory and asymptotic properties of solutions of even order neutral difference equations. J. Differ. Equ. Appl. 15, 1077-1084 (2009)

12. Migda, M, Zhang, G: Monotone solutions of neutral difference equations of odd order. J. Differ. Equ. Appl. 10, 691-703 (2004)

13. Zhou, Y, Zhang, BG: Existence of nonoscillatory solutions of higher-order neutral delay difference equations with variable coefficients. Comput. Math. Appl. 45, 991-1000 (2003)

14. Saker, SH: New oscillation criteria for third order nonlinear neutral difference equations. Math. Slovaca 61, 579-600 (2011)

15. Saker, SH: Oscillation of a certain class of third order nonlinear difference equations. Bull. Malays. Math. Sci. Soc. 35, 651-669 (2012)

16. Andruch-Sobiło, A, Migda, M: Bounded solutions of third order nonlinear difference equations. Rocky Mt. J. Math. 36, 23-34 (2006)

17. Došlá, Z, Kobza, A: On third-order linear difference equations involving quasi-differences. Adv. Differ. Equ. 2006 Article ID 65652 (2006). doi:10.1155/ADE/2006/65652

18. Graef, J, Thandapani, E: Oscillatory and asymptotic behaviour of solutions of third-order delay difference equations. Funkc. Ekvacioj 42, 355-369 (1999)

19. Liu, Z, Wang, L, Kimb, G, Kang, S: Existence of uncountably many bounded positive solutions for a third order nonlinear neutral delay difference equation. Comput. Math. Appl. 60, 2399-2416 (2010)

20. Saker, SH: Oscillation Theory of Delay Differential and Difference Equations: Second and Third Orders. Verlag dr. Müller, Saarbrücken (2010)

21. Zhang, BG, Cheng, SS: On a class of nonlinear difference equations. J. Differ. Equ. Appl. 1, 391-411 (1995)

22. Yan, J, Liu, B: Oscillatory and asymptotic behavior of fourth order nonlinear difference equations. Acta Math. Sin. 13, 105-115 (1997)

23. Graef, JR, Thandapani, E: Oscillatory and asymptotic behavior of fourth order nonlinear delay difference equations. Fasc. Math. 31, 23-36 (2001)

24. Migda, M, Musielak, A, Schmeidel, E: On a class of fourth order nonlinear difference equations. Adv. Differ. Equ. 2004 23-36 (2004)

25. Migda, M, Schmeidel, E: Asymptotic properties of fourth order nonlinear difference equations. Math. Comput. Model. 39, 1203-1211 (2004)

26. Cheng, SS, Patula, WT: An existence theorem for a nonlinear difference equation. Nonlinear Anal. 20, 193-203 (1993)

27. Győri, I, Ladas, G: Oscillation Theory of Delay Differential Equations with Applications. Clarendon, Oxford (1991)

28. Agarwal, RP: Difference Equations and Inequalities: Theory, Methods, and Applications. Dekker, New York (1992)

29. Migda, M: Oscillation and nonoscillation results for higher order nonlinear difference equations. Fields Inst. Commun. 42, 285-294 (2004)

30. Hasanbulli, M, Rogovchenko, YV: Asymptotic behavior nonoscillatory solutions to $n$-th order nonlinear neutral differential equations. Nonlinear Anal. 69, 1208-1218 (2008)

31. Migda, J: Asymptotically polynomial solutions of difference equations. Adv. Differ. Equ. 2013, 92 (2013)

32. Migda, J: Approximative solutions of difference equations. Electron. J. Qual. Theory Differ. Equ. 2014, 13 (2014)

33. Migda, J, Migda, M: On the asymptotic behavior of solutions of higher order nonlinear difference equations. Nonlinear Anal. 47, 4687-4695 (2001)

34. Philos, CG, Purnaras, IK, Tsamatos, PC: Asymptotic to polynomials solutions for nonlinear differential equations. Nonlinear Anal. 59, 1157-1179 (2004)

\section{Submit your manuscript to a SpringerOpen ${ }^{\odot}$ journal and benefit from:}

- Convenient online submission

Rigorous peer review

- Immediate publication on acceptance

- Open access: articles freely available online

- High visibility within the field

- Retaining the copyright to your article

Submit your next manuscript at $\boldsymbol{s p r i n g e r o p e n . c o m ~}$ 